\title{
Acute Adrenal Insufficiency Precipitated by the Discontinuation of a Betamethasone and Dextrochlorpheniramine Combination: The Diagnostic Utility of an Echocardiographic Assessment of Systemic Vascular Resistance
}

Jun Mukai ${ }^{1}$, Shumpei Mori ${ }^{1}$, Yukiko Katsumori-Yoshimura ${ }^{1}$, Ryo Takeshige ${ }^{1}$, Tokiko Tabata $^{1}$, Hiroshi Imada ${ }^{1}$, Hiroyuki Shimoura ${ }^{1}$, Hachidai Takahashi ${ }^{1}$, Yutaka Takahashi ${ }^{2}$ and Ken-ichi Hirata ${ }^{1}$

\begin{abstract}
:
A 72-year-old woman with primary biliary cholangitis was admitted to our hospital with heart failure with a preserved ejection fraction. An accidental right ventricular perforation that occurred during an endomyocardial biopsy precipitated cardiogenic shock. Despite successful surgical treatment, she demonstrated progressive hemodynamic deterioration, which was resistant to the administration of high-dose catecholamines. She was diagnosed with acute adrenal insufficiency, which was attributed to the discontinuation of Celestamine ${ }^{\circledR}$ (betamethasone/dextrochlorpheniramine combination) just after the perforation. Prompt intravenous administration of hydrocortisone $(150 \mathrm{mg} / \mathrm{day})$ led to hemodynamic stabilization. The serial noninvasive assessment of systemic vascular resistance using transthoracic echocardiography was instrumental in detecting acute adrenal insufficiency in this case.
\end{abstract}

Key words: acute adrenal insufficiency, betamethasone, celestamine, primary biliary cholangitis, systemic vascular resistance

(Intern Med 58: 2045-2049, 2019)

(DOI: 10.2169/internalmedicine.2502-18)

\section{Introduction}

Chronic glucocorticoid administration represents a widelyprescribed therapy for several diseases in consideration of both its anti-inflammatory and immunosuppressive activity (1). Almost $1 \%$ of the adult population have been exposed to oral glucocorticoids (2). However, considering the wide use of synthetic glucocorticoids, many patients could potentially be at risk of developing adrenal insufficiency particularly after the withdrawal of glucocorticoids (1). Approximately $6 \%$ of hospitalized patients may therefore have glucocorticoid-induced adrenal insufficiency (3).

Celestamine ${ }^{\circledR}$ (TAKATA Pharmaceutical, Saitama, Japan) is a steroid preparation containing betamethasone (one of the most used synthetic glucocorticoids) and dextrochlorpheniramine maleate as its active ingredients, which is oftenly used for allergic diseases. However, an inadequate awareness of the dose/drug information may lead to the injudicious (and often prolonged) administration of this medication.

The presence of unexplained catecholamine-refractory hypotension is a characteristic finding in patients with acute adrenal insufficiency (4). In the diagnosis of acute adrenal insufficiency, however, few reports have demonstrated the utility of thransthoracic echocardiogrpahy, which is the fistline diagnostic tool for the noninvasive and early differentiation of shock.

We herein report a case of acute adrenal insufficiency triggered by the abrupt discontinuation of 2 Celestamine $^{\circledR}$

${ }^{1}$ Division of Cardiovascular Medicine, Department of Internal Medicine, Kobe University Graduate School of Medicine, Japan and ${ }^{2}$ Division of Diabetes and Endocrinology, Department of Internal Medicine, Kobe University Graduate School of Medicine, Japan

Received: December 8, 2018; Accepted: January 30, 2019; Advance Publication by J-STAGE: April 17, 2019

Correspondence to Dr. Shumpei Mori, shumpei_8@ hotmail.com 
Table. Laboratory Data on Admission.

\begin{tabular}{|c|c|c|c|}
\hline Peripheral blood & & iochemistry & \\
\hline White blood cells & $6,600 / \mu \mathrm{L}$ & Sodium & $136 \mathrm{mEq} / \mathrm{L}$ \\
\hline Red blood cells & $285 \times 10^{4} / \mu \mathrm{L}$ & Potassium & $4.0 \mathrm{mEq} / \mathrm{L}$ \\
\hline Hemoglobin & $9.0 \mathrm{~g} / \mathrm{dL}$ & Chloride & $99 \mathrm{mEq} / \mathrm{L}$ \\
\hline Hematocrit & $29.2 \%$ & Calcium & $8.6 \mathrm{mg} / \mathrm{dL}$ \\
\hline Platelets & $24.4 \times 10^{4} / \mu \mathrm{L}$ & Urea nitrogen & $15.1 \mathrm{mg} / \mathrm{dL}$ \\
\hline Neutrophil & $54.7 \%$ & Serum creatinine & $0.69 \mathrm{mg} / \mathrm{dL}$ \\
\hline Eosinophil & $0.5 \%$ & Estimated glomerular filtration rate & $63.1 \mathrm{~mL} / \mathrm{min} / 1.73 \mathrm{~m}^{2}$ \\
\hline Monocyte & $4.8 \%$ & Total protein & $6.2 \mathrm{~g} / \mathrm{dL}$ \\
\hline \multirow[t]{2}{*}{ Lymphocyte } & $39.2 \%$ & Serum albumin & $2.6 \mathrm{~g} / \mathrm{dL}$ \\
\hline & & Asparate aminotransferase & $21 \mathrm{IU} / \mathrm{L}$ \\
\hline Blood coagulation & & Alanine aminotransferase & $6 \mathrm{IU} / \mathrm{L}$ \\
\hline Actipated partial thromboplastin & $29.5 \mathrm{sec}$ & Alkaline phosphate & $381 \mathrm{IU} / \mathrm{L}$ \\
\hline Prothrombin time-international normalized ratio & 1.1 & Creatine kinase & $113 \mathrm{IU} / \mathrm{L}$ \\
\hline Prothrombin time $\%$ & $81.7 \%$ & Creatine kinase MB & $<4 \mathrm{IU} / \mathrm{L}$ \\
\hline \multirow[t]{2}{*}{ D-dimer } & $0.9 \mu \mathrm{g} / \mathrm{mL}$ & Lactate dehydrogenase & $134 \mathrm{IU} / \mathrm{L}$ \\
\hline & & Aldolase & $6.1 \mathrm{IU} / \mathrm{L}$ \\
\hline Immunology & & Total cholesterol & $254 \mathrm{mg} / \mathrm{dL}$ \\
\hline Anti-mitochondrial antibody & $\times 320$ & Triglycerides & $217 \mathrm{mg} / \mathrm{dL}$ \\
\hline \multirow[t]{3}{*}{ Anti-mitochondrial M2 antibody } & $240 \mathrm{U} / \mathrm{L}$ & Brain natriuretic peptide & $251 \mathrm{pg} / \mathrm{mL}$ \\
\hline & & Glucose & $75 \mathrm{mg} / \mathrm{dL}$ \\
\hline & & Hemoglobin A1c & $5.3 \%$ \\
\hline
\end{tabular}

tablets in a patient with a history of long-term ( $>13$ years) daily Celestamine ${ }^{\circledR}$ use. Furthermore, this case highlights the importance of a serial echocardiographic assessment of systemic vascular resistance to diagnose patients with a strong index of clnical suspicion for this condition and to follow the clinical course of such patients with acute adrenal insufficiency.

\section{Case Report}

A 72-year-old lean woman (body mass index $14.3 \mathrm{~kg} / \mathrm{m}^{2}$ ) with primary biliary cholangitis was admitted to our hospital with general fatigue and dyspnea on effort. She had a history of a lumbar vertebral compression fracture secondary to osteoporosis and heart failure due to tachycardia-induced cardiomyopathy. Additionally, she reported a history of persistent atrial fibrillation with a rapid ventricular response, which had relapsed even after undergoing radiofrequency catheter ablation. Paroxysmal atrial flutter and sinus bradycardia had been reported as well. She denied a family history of cardiac disease. Upon admission, her heart failure symptoms were classified as New York Heart Association Class III. Her daily medications included bisoprolol fumarate $(5 \mathrm{mg})$, enalapril maleate $(1.25 \mathrm{mg})$, rivaroxaban (10 $\mathrm{mg}$ ), ursodeoxycholic acid $(900 \mathrm{mg}$ ), and 2 tablets of Celestamine $^{\circledR}$ (combination of betamethasone $0.25 \mathrm{mg}$ and dextrochlorpheniramine $5 \mathrm{mg}$ ). Physical examination revealed marked sarcopenia. Her blood pressure was 90/42 mmHg with a regular pulse rate of 60 beats/min. No cardiac murmur or respiratory crackles were audible. Laboratory tests indicated normocytic anemia. Her serum brain natriuretic peptide level was elevated to $251 \mathrm{pg} / \mathrm{mL}$. The serum level of anti-mitochondrial antibody M2 was elevated to $240 \mathrm{U} / \mathrm{mL}$ (Table). Chest radiography showed no cardiomegaly (cardiothoracic ratio $47 \%$ ) and no evidence of congestion or pleural effusion. Her electrocardiogram showed a normal sinus rhythm with $\mathrm{P}$-waves showing an extremely low voltage and T-wave inversion in the precordial leads. Transthoracic echocardiography showed mild generalized hypokinesis of the left ventricle (ejection fraction 52\%) concomitant with bilateral atrial dilatation predominantly affecting the right atrium. No left ventricular dilatation and/or hypertrophy or pericardial effusion was observed. Transmitral Doppler inflow patterns showed pseudo-normalization (E/A ratio 1.02), and mitral annulus velocity by tissue Doppler imaging suggested impaired left ventricular relaxation (e' $5.3 \mathrm{~cm} / \mathrm{s}$ ). Coronary angiography showed intact coronary arteries. Right heart catheterization after the treatment of heart failure showed the mean pulmonary capillary wedge pressure to be $6 \mathrm{mmHg}$, the pulmonary artery pressure was $22 / 8 \mathrm{mmHg}$, and the mean right atrial pressure was $6 \mathrm{mmHg}$. The cardiac index measured using the calculated-Fick method was reduced to $1.73 \mathrm{~L} / \mathrm{min} / \mathrm{m}^{2}$. Based on the electrocardiographic findings (multiple supraventricular arrhythmias, the coincidence of tachy- and bradyarrhythmias, and extremely low atrial deflections) and echocardiographic findings (bilateral atrial dilatation with generalized mild hypokinesis of the left ventricle with diastolic dysfunction), we suspected antimitochondrial M2 antibody-associated cardiomyopathy with possible atrial and ventricular involvements (5-7). An endomyocardial biopsy was then performed on the right-sided ventricular septum. Unfortunately, accidental right ventricular perforation occurred during the biopsy procedure, and the patient developed cardiogenic shock that was refractory 


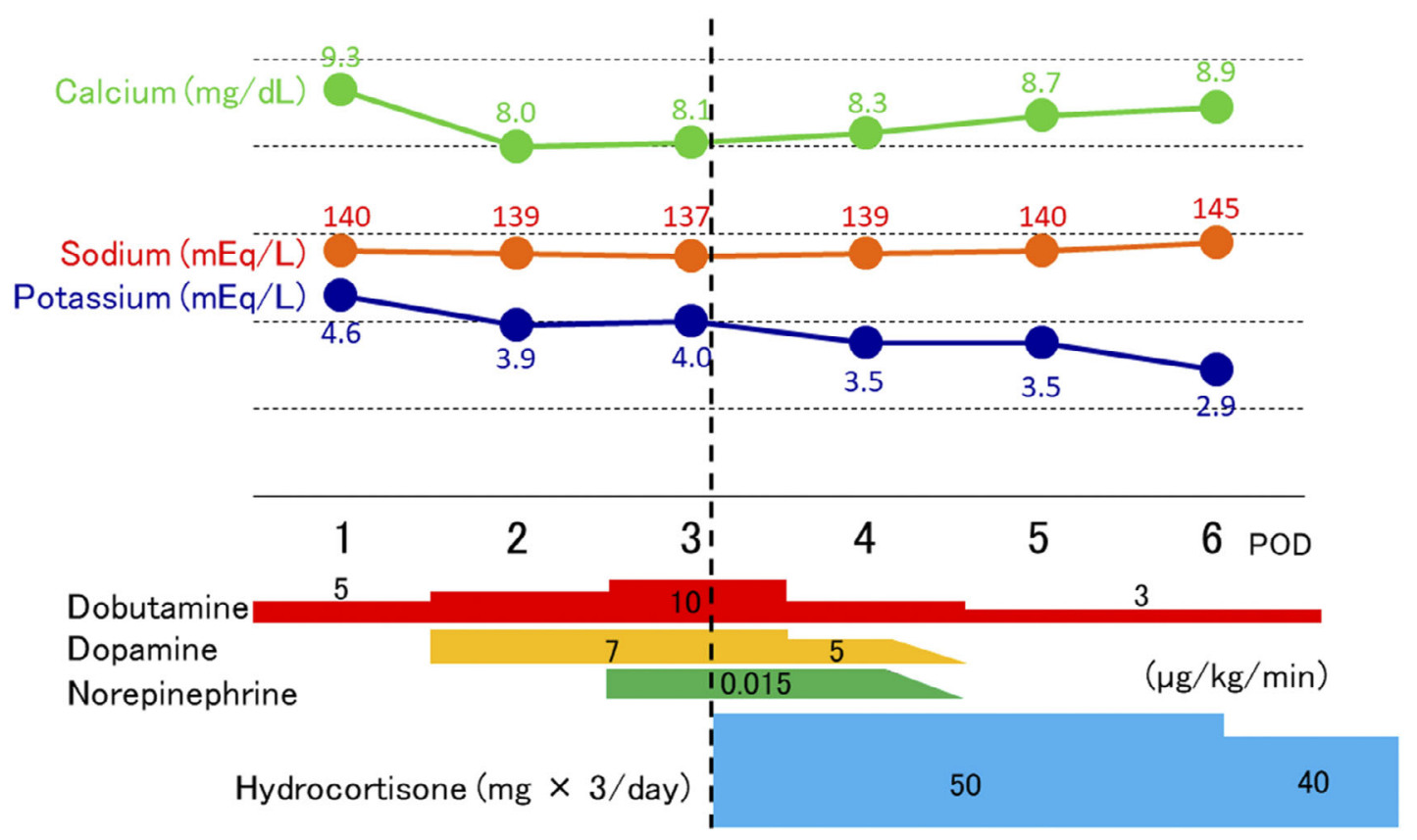

Figure 1. Clinical course of our patient after right ventricular perforation (1). It was difficult to suspect acute adrenal insufficiency based on the serum electrolyte levels. POD: post-operative day

to pericardiocentesis. She underwent surgical closure using percutaneous cardiopulmonary support. However, the development of hemodynamic instability immediately after the successful surgical closure of the perforation necessitated transfer to the cardiac care unit along with the administration of $5 \mu \mathrm{g} / \mathrm{kg} / \mathrm{min}$ of dobutamine. She was intubated, and all medications were temporarily discontinued. Transthoracic echocardiography performed postoperatively in the cardiac care unit showed findings compatible with biventricular Takotsubo cardiomyopathy, which led us to consider this condition as the primary cause of hemodynamic instability. A progressive reduction in her blood pressure and her daily urine output was observed, although daily echocardiographic assessment showed no deterioration and in fact, a rapid improvement in biventricular Takotsubo cardiomyopathy. The patient's fluid status did not indicate hypovolemia as evident by central venous pressure consistently $>12 \mathrm{mmHg}$. Echocardiographic evaluation of the inferior caval vein also excluded hypovolemic shock, as shown by its diameter $>18$ $\mathrm{mm}$, constantly. The serum electrolyte (sodium and potassium) as well as calcium and lactate levels did not show significant variations (Fig. 1). She did not show any high-grade fever or other clinical findings indicating septic shock. Based on the serial monitoring of the cardiac output by daily transthoracic echocardiography, systemic vascular resistance was calculated from the following formula: (Mean arterial pressure - mean central venous pressure) / cardiac output $\times 80$ (dynes $\left.\cdot \mathrm{sec} \cdot \mathrm{cm}^{-5}\right)$, which indicated a progressive reduction in vascular tone (Fig. 2). On day 3 after the perforation, her serum glucose levels were relatively low $(77 \mathrm{mg}$ / dL) despite the prior initiation of intravenous hyperalimentation. Following a further reduction in the systemic vascular resistance $\left(740\right.$ dynes $\cdot \sec \cdot \mathrm{cm}^{-5}$ ) on day 3 , we suspected acute adrenal insufficiency, which was attributable to the discontinuation of Celestamine ${ }^{\circledR}$. We confirmed her history of long-term (>13 years) use of 2 tablets of Celestamine ${ }^{\circledR}$ (dose equivalent to $5 \mathrm{mg}$ of prednisolone). Therefore, we decided to treat her with the intravenous administration of hydrocortisone $(150 \mathrm{mg} / \mathrm{day})$ for suspected acute adrenal insufficiency immediately after the submission of hormonal blood test because of emergency status. Interestingly, hydrocortisone treatment rapidly led to complete hemodynamic stabilization and prompt normalization of the systemic vascular resistance (Fig. 2). Pretreatment level of her serum adrenocorticotropic hormone and cortisol were $167.9 \mathrm{pg} / \mathrm{mL}$ and 32.6 $\mu \mathrm{g} / \mathrm{dL}$, respectively, which were revealed after the initiation of treatment. Her further clinical course was uneventful, and the intravenous hydrocortisone dose was subsequently tapered and eventually replaced with oral hydrocortisone (10 $\mathrm{mg}$ /day) prescribed as an outpatient.

\section{Discussion}

Celestamine $^{\circledR}$ is a combination preparation containing betamethasone $(0.25 \mathrm{mg})$ and dextrochlorpheniramine $(2 \mathrm{mg})$ per tablet. Conventionally, it is prescribed to treat the acute phase of any allergic inflammation, including urticaria, eczema, dermatitis, drug eruptions, and/or allergic rhinitis (8). Betamethasone is a synthetic long-acting glucocorticoid. The glucocorticoid dose in Celestamine ${ }^{\circledR}$ is equivalent to $2.5 \mathrm{mg}$ of prednisolone per tablet. Thus, drug information alerts to unthinking chronic administration to avoid iatrogenic Cushing's syndrome. Withdrawal from glucocorticoid therapy (usually long-term) is a common cause of adrenal insufficiency (9). Thus, a dose reduction or complete discontinuation of long-term Celestamine ${ }^{\circledR}$ can precipitate secondary 


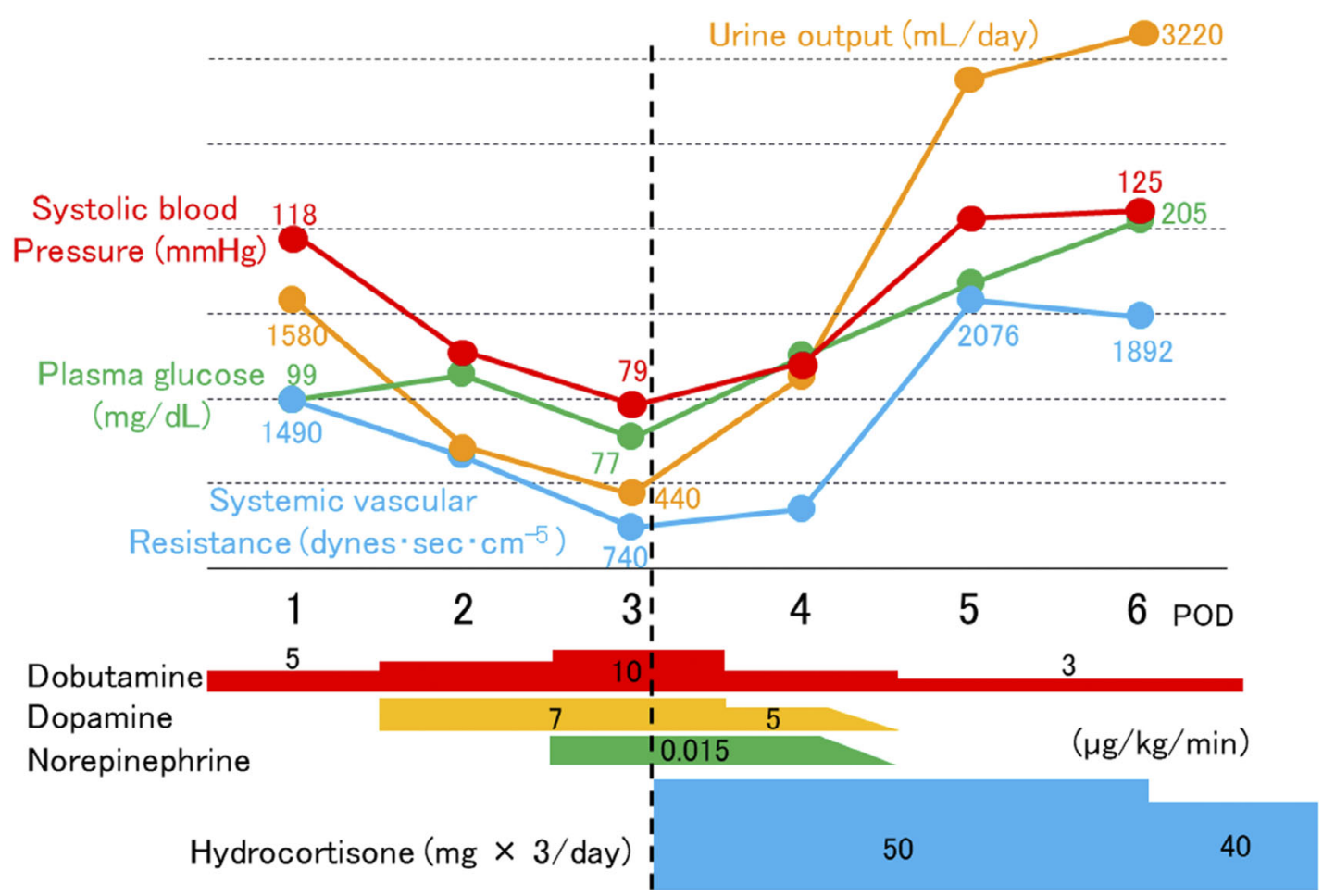

Figure 2. Clinical course of our patient after right ventricular perforation (2). A progressive reduction in systemic vascular resistance, as well as features of catecholamine-refractory hypotension and relative hypoglycemia served as indicators of suspected acute adrenal insufficiency. POD: post-operative day

acute adrenal insufficiency due to the suppression of the hypothalamic-pituitary-adrenal axis. Thus, patients should be carefully monitored, and the reactivation of the basic disease also needs evaluation.

The accurate prevalence rates of adrenal insufficiency associated with glucocorticoid therapy are unknown; however, approximately $6 \%$ of hospitalized patients may have glucocorticoid-induced adrenal insufficiency (3), and presumably $\geq 30 \%$ of patients taking glucocorticoids are affected (9). The glucocorticoid dose or the duration of treatment that can affect adrenal function after glucocorticoid withdrawal cannot be accurately predicted owing to variations between patients $(9,10)$. In the present case, Celestamine ${ }^{\circledR}$ equivalent to a daily dose of $5 \mathrm{mg}$ of prednisolone had been administered for $\geq 13$ years, which was sufficient to inhibit the patient's endogenous hypothalamic-pituitaryadrenal axis.

The symptoms and signs of adrenal insufficiency are nonspecific; thus, the diagnosis is often delayed (9). Clinically, patients present with fever, confusion, nausea, fluid resuscitation- and catecholamine-refractory hypotension, hypoglycemia, hyponatremia/hyperkalemia, eosinophilia, and metabolic lactic acidosis $(4,11-13)$. Serial observations of these features following the triggered event are important to suspect acute adrenal insufficiency. As was observed in this case without typical hyponatremia nor hyperkalemia, however, these findings may be masked or modified in an intensive care setting secondary to daily fluid replacement measures, including intravenous hyperalimentation and the use of several inotropic and vasoactive agents. In addition to the typical features characterizing catecholamine-refractory hypotension and relative hypoglycemia that progressed despite hyperalimentation, the serial noninvasive assessment of systemic vascular resistance using transthoracic echocardiography was instrumental in detecting acute adrenal insufficiency in this patient (Fig. 2). The accurate estimation of the cardiac output, right atrial pressure, and systemic vascular resistance is crucial for primary differential diagnosis of cardiogenic shock, hypovolemic shock, and shock related to loss in systemic vascular resistance, including acute adrenal insufficiency and anaphylactic shock. The noninvasive, prompt, and serial assessment of all of these features can be readily achieved using echocardiography; thus, it is fundamental tool in the setting of critical care for cardiologists. These values can also be assessed via pulmonary artery catheterization; however, critical care management via daily bedside transthoracic echocardiography is a non-invasive approach that is considered to be better than the long-term insertion of a pulmonary artery catheter (14).

A single test cannot accurately diagnose adrenal insufficiency in a patient requiring intensive care for critical illness-related adrenal insufficiency $(9,13)$. Usually, clinicians use a random serum cortisol level of $<10 \mu \mathrm{g} / \mathrm{dL}$ as a reference (13), and a serum cortisol level of $>25 \mu \mathrm{g} / \mathrm{dL}$ rules out adrenal insufficiency. However, the safe and accurate cut-off value still remains controversial (4). Notably, a serum cortisol level within the normal range does not rule out adrenal insufficiency in an acutely ill patient $(4,11-13)$ be- 
cause the corticotropic axis is highly activated in such patients. The serum cortisol levels are strongly associated with illness severity and show the highest levels in patients with the highest mortality (11). In the present case, although the serum cortisol level was $>25 \mu \mathrm{g} / \mathrm{dL}$, the history of long-term use of betamethasone and prompt hemodynamic stabilization after corticosteroid administration strongly supported the diagnosis of acute adrenal insufficiency. Thus, a relatively high cortisol value may indicate a critical situation as supported by the compensatory increase in adrenocorticotropic hormone level. In patients with newly diagnosed (or suspected) adrenal insufficiency, baseline blood samples should be drawn immediately to measure cortisol and adrenocorticotropic hormone levels before hydrocortisone administration. However, this is a critical condition; thus, a timeconsuming diagnostic workup should be avoided to ensure prompt initiation of treatment even before diagnostic confirmatory testing is completed $(4,11,12)$. A diagnostic delay may result in potentially fatal consequences following the failure of physiological compensation to the invasive stress. Critically ill patients with adrenal insufficiency should receive intravenous infusions of high-dose hydrocortisone (100 $\mathrm{mg}$ as a bolus dose followed by infusions of 100-200 mg administered over 24 hours) $(4,11,13)$.

On the basis of its brand name, it is difficult to deduce that Celestamine ${ }^{\circledR}$ tablet contains a glucocorticoid dose equivalent to $2.5 \mathrm{mg}$ of prednisolone. This fact perhaps contributes to the injudicious and often long-term prescription of Celestamine ${ }^{\circledR}$ from non-specialists, particularly because it is highly effective for allergic symptoms and therefore a popular drug in clinical practice. Obtaining a thorough medication history is important in patients undergoing surgery or patients in emergency settings, particularly focusing on the use of steroids (15). Unfortunately, we did not focus on the medication history in our patient, particularly her history of long-term Celestamine ${ }^{\circledR}$ use. Thus, we did not initially suspect acute adrenal insufficiency in this patient.

In conclusion, this case highlights the importance of promptly identifying the potential risk of long-term Celestamine ${ }^{\circledR}$ use. In our view, this case report is considered to be beneficial for clinicians in Japan by emphasizing the risks of the injudicious use of Celestamine ${ }^{\circledR}$.

The authors state that they have no Conflict of Interest (COI).

\section{References}

1. Paragliola RM, Papi G, Pontecorvi A, Corsello SM. Treatment with synthetic glucocorticoids and the hypothalamus-pituitaryadrenal axis. Int J Mol Sci 2017.

2. Fardet L, Petersen I, Nazareth I. Prevalence of long-term oral glucocorticoid prescriptions in the UK over the past 20 years. Rheumatology (Oxford) 50: 1982-1990, 2011.

3. Smans LC, Van der Valk ES, Hermus AR, Zelissen PM. Incidence of adrenal crisis in patients with adrenal insufficiency. Clin Endocrinol (Oxf) 84: 17-22, 2016.

4. Oelkers W. Adrenal insufficiency. N Engl J Med 335: 1206-1212, 1996.

5. Maeda MH, Tsuji S, Shimizu J. Inflammatory myopathies associated with anti-mitochondrial antibodies. Brain 135: 1767-1777, 2012.

6. Matsumoto K, Tanaka H, Yamana S, et al. Successful steroid therapy for heart failure due to myocarditis associated with primary biliary cirrhosis. Can J Cardiol 28: 515. e3-e6, 2012.

7. Konishi H, Fukuzawa K, Mori S, et al. Anti-mitochondrial M2 antibodies enhance the risk of supraventricular arrhythmias in patients with elevated hepatobiliary enzyme levels. Intern Med 56: 1771-1779, 2017.

8. Noferi A, Mancuso A. Association of betamethasone and dextrochlorpheniramine (Celestamine) in the symptomatic treatment of pollinosis. Folia Allergol (Roma) 19: 53-56, 1972.

9. Guerrero Pérez F, Marengo AP, Villabona Artero C. The unresolved riddle of glucocorticoid withdrawal. J Endocrinol Invest 40: 1175-1181, 2017.

10. Dinsen S, Baslund B, Klose M, et al. Why glucocorticoid withdrawal may sometimes be as dangerous as the treatment itself. Eur J Intern Med 24: 714-720, 2013.

11. Arlt W, Allolio B. Adrenal insufficiency. Lancet 361: 1881-1893, 2003.

12. Fares AB, Santos RA. Conduct protocol in emergency: Acute adrenal insufficiency. Rev Assoc Med Bras (1992) 62: 728-734, 2016.

13. Annane D, Pastores SM, Rochwerg B, et al. Guidelines for the Diagnosis and Management of Critical Illness-Related Corticosteroid Insufficiency (CIRCI) in Critically Ill Patients (Part I): Society of Critical Care Medicine (SCCM) and European Society of Intensive Care Medicine (ESICM) 2017. Crit Care Med 45: 2078-2088, 2017.

14. Sandham JD, Hull RD, Brant RF, et al; Canadian Critical Care Clinical Trials Group. A randomized, controlled trial of the use of pulmonary-artery catheters in high-risk surgical patients. N Engl J Med 348: 5-14, 2003.

15. Robati S, Shahid MK, Vella A, Rang S. Importance of a thorough drug history in presurgical patients. BMJ Case Rep, 2014.

The Internal Medicine is an Open Access journal distributed under the Creative Commons Attribution-NonCommercial-NoDerivatives 4.0 International License. To view the details of this license, please visit (https://creativecommons.org/licenses/ by-nc-nd/4.0/).

(C) 2019 The Japanese Society of Internal Medicine

Intern Med 58: 2045-2049, 2019 